\title{
Article \\ Cumulative Residual Tsallis Entropy-Based Test of Uniformity and Some New Findings
}

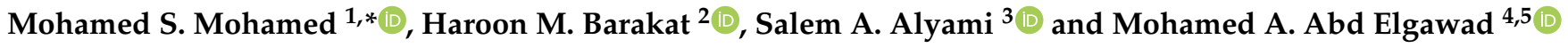 \\ 1 Department of Mathematics, Faculty of Education, Ain Shams University, Cairo 11341, Egypt \\ 2 Department of Mathematics, Faculty of Science, Zagazig University, Zagazig 44519, Egypt; \\ hbarakat@zu.edu.eg \\ 3 Department of Mathematics and Statistics, Faculty of Science, Imam Mohammad Ibn Saud Islamic University \\ (IMSIU), Riyadh 13318, Saudi Arabia; saalyami@imamu.edu.sa \\ 4 Department of Mathematics, Faculty of Science, Benha University, Benha 13518, Egypt; \\ mohamed.abdelgwad@fsc.bu.edu.eg \\ 5 School of Computer Science and Technology, Wuhan University of Technology, Wuhan 430070, China \\ * Correspondence: mohamed.said@edu.asu.edu.eg
}

check for

updates

Citation: Mohamed, M.S.; Barakat, H.M.; Alyami, S.A.; Abd Elgawad, M.A. Cumulative Residual Tsallis Entropy-Based Test of Uniformity and Some New Findings.

Mathematics 2022, 10, 771. https:// doi.org/10.3390/math10050771

Academic Editors: Carlos Agra Coelho and Tatjana von Rosen

Received: 27 January 2022

Accepted: 23 February 2022

Published: 28 February 2022

Publisher's Note: MDPI stays neutral with regard to jurisdictional claims in published maps and institutional affiliations.

Copyright: (c) 2022 by the authors. Licensee MDPI, Basel, Switzerland. This article is an open access article distributed under the terms and conditions of the Creative Commons Attribution (CC BY) license (https:// creativecommons.org/licenses/by/ $4.0 /)$.

\begin{abstract}
The Tsallis entropy is an extension of the Shannon entropy and is used extensively in physics. The cumulative residual Tsallis entropy, which is a generalization of the Tsallis entropy, plays an important role in the measurement uncertainty of random variables and has simple relationships with other important information and reliability measures. In this paper, some novel properties of the cumulative residual Tsallis entropy are disclosed. Moreover, this entropy measure is applied to testing the uniformity, where the limit distribution and an approximation of the distribution of the test statistic are derived. In addition, the property of stability is discussed. Furthermore, the percentage points and power against seven alternative distributions of this test statistic are presented. Finally, to compare the power of the suggested test with that of other tests of uniformity, a simulation study is conducted.
\end{abstract}

Keywords: cumulative residual Tsallis entropy; stability; empirical cumulative distribution function; testing uniformity; Monte Carlo method; test power

MSC: 62G05; 62G30; 94A15; 94A17

\section{Introduction}

The classical measure of uncertainty in a discrete distribution (Shannon [1]) has been used in many areas, such as computer science [2], communication theory [2], the physical and chemical fields [3], fuzzy sets [4], and finance [5,6]. A straightforward extension of the discrete case to continuous distributions based on a probability density function (PDF) $f_{X}($.$) of a continuous random variable (\mathrm{RV}) X$, called differential entropy, reads (cf. [7])

$$
S(X)=-\mathrm{E}\left(\log f_{X}(X)\right)=-\int_{-\infty}^{\infty} f_{X}(x) \log f_{X}(x) d x .
$$

Many generalizations of the Shannon entropy have been published by inserting some additional parameters, making these generalizations more responsive to diverse shapes of probability distributions. Rao et al. [7] (see also, Wang et al. [8]) suggested a non-negative measure of uncertainty and referred to it as the cumulative residual entropy (CRE). This suggested measure is obtained by replacing the PDF $f_{X}(x)$ in (1) by the survival function $\bar{F}_{X}(x):=P(X>x)=1-F_{X}(x)$. Thus, for any continuous $\mathrm{RV} X$ with a cumulative distribution function (CDF) $F_{X}(x)$, the CRE is specified by

$$
\operatorname{CRE}(F)=-\int_{0}^{\infty} \bar{F}_{|X|}(x) \log \left(\bar{F}_{|X|}(x)\right) d x
$$


where $\bar{F}_{|X|}(x)=P(|X|>x)$.

The Tsallis entropy of order $\theta$ is a generalization of the Shannon entropy that was first given by Havrda and Charvat [9]. Then, Tsallis [10] used its properties and placed it in a physical context. This measure is defined for any continuous RV X as

$$
T_{\theta}(X)=\frac{1}{\theta-1} \mathrm{E}\left(1-\left(f_{X}(X)\right)^{\theta-1}\right)=\frac{1}{\theta-1}\left(1-\int_{-\infty}^{\infty}\left(f_{X}(x)\right)^{\theta} d x\right),
$$

where $1 \neq \theta>0$. Clearly, as $\theta \rightarrow 1, T_{\theta}(X) \rightarrow S(X)$.

Motivated by the wide applicability of the Tsallis entropy, Sati and Gupta [11] proposed the cumulative residual Tsallis entropy (CRTE) of order $\theta$, which is given by

$$
C T_{\theta}^{*}(X)=\frac{1}{\theta-1}\left(1-\int_{0}^{\infty}\left(\bar{F}_{|X|}(x)\right)^{\theta} d x\right), 1 \neq \theta>0 .
$$

Rajesh and Sunoj [12] introduced an alternate measure of CRTE of order $\theta$, which possesses certain interesting properties with $C T_{\theta}^{*}(X)$, as

$$
C T_{\theta}(X)=C T_{\theta}(F)=\frac{1}{\theta-1}\left(\int_{0}^{\infty}\left(\bar{F}_{|X|}(x)-\left(\bar{F}_{|X|}(x)\right)^{\theta}\right) d x\right), 1 \neq \theta>0 ;
$$

when $\theta \rightarrow 1$, then $C T_{\theta}(X) \rightarrow C R E(F)$ but $C T_{\theta}^{*}(X) \nrightarrow C R E(F)$, and $C T_{\theta}(X)=C T_{\theta}^{*}(X)+$ $\frac{\mathrm{E}(X)-1}{\theta-1}$, for more details see Mohamed [13]. In this work we focus only on the measure (2).

Rajesh and Sunoj [12] detected several eminent features of the CRTE (2). For example, the CRTE has more interesting mathematical features than the CRE, it can be easily estimated from sample data, and these estimates asymptotically converge to the true values. Moreover, the CRTE handles the information in residual life. For the standard uniform distribution, denoted by $U(0,1)$, Rajesh and Sunoj [12] determined the value of the CRTE, which is $\frac{1}{2(\theta+1)}$. The literature teems with several results of the Shannon entropy and its related measures. Interested readers may refer to [14-18] for the Shannon entropy, Kullback-Leibler divergence, and Fisher information number; $[19,20]$ for fractional cumulative residual entropy and cumulative residual entropy, respectively; [13,21-24] for the Tsallis entropy and its related measures; and finally $[25,26]$ for the extropy and Rényi entropy and its applications, respectively.

Stephens [27] provided a useful guide to goodness-of-fit tests using statistics based on the empirical CDF. Furthermore, power comparisons of several uniformity tests were performed in [27]. The power attributes of an entropy-based test when employed for measuring uniformity were examined by Dudewicz and Van der Meulen [28]. Furthermore, Dudewicz and Van der Meulen [28] demonstrated that the entropy-based test has good power qualities for various alternatives by comparing it to other uniformity tests. Noughabi [29] developed a test for uniformity based on the CRE and studied some of its features. In addition, he compared the percentage points and power of seven alternative distributions. Mohamed et al. [30] used the fractional and weighted CRE measures to test the uniformity.

In this paper, we study the CRTE (2) for testing uniformity. The outcome of a simulation study reveals that the test under CRTE is competing with the test based on CRE in terms of power. In addition, some interesting statistical properties of the CRTE are revealed. We also use the Monte Carlo method via simulation and normality asymptotic, as well as the beta approximation, to derive the percentage points under the CRTE. In addition, the CRTE and other tests are compared in terms of power analysis.

\section{Work Motivation}

The Tsallis entropy of order $\theta$, which was introduced by Tsallis [10], plays an important role in the measurement uncertainty of RVs and leads nonextensive statistics. The Tsallis entropy is the basis of the so-called nonextensive statistical mechanics, which generalizes 
the Boltzmann-Gibbs theory (cf. [31]). Tsallis statistics have found applications in a wide range of phenomena in diverse disciplines such as physics, chemistry, biology, medicine, economics, geophysics, etc. For example, Cartwright [32] proposed applications of the Tsallis entropy in various fields, such as describing the fluctuation of the magnetic field in the solar wind and signs of breast cancer in mammograms. Sati and Gupta [11] introduced a cumulative residual Tsallis entropy of order $\theta$ and studied its various properties in the context of reliability modeling. After one year, Rajesh and Sunoj [12] introduced an alternate measure of CRTE (defined by (2)) and studied its properties. Unlike the CRTE of Sati and Gupta [11], the proposed measure had some additional features and had simple relationships with other important information and reliability measures.

There are many different types of probability distributions, and the uniform distribution is perhaps the simplest of them all. For a continuous distribution, the uniform distribution defines equal probability over a given range. As a result, it is valuable as a reference distribution. Random number generation is one of the most important uses of uniform distribution. Moreover, in the field of economics, usually, demand and replenishment may not follow the expected normal distribution. As a result, different distribution models are employed to better anticipate probabilities and trends. According to Wanke [33], uniform distribution is more effective when evaluating lead-time for inventory management at the beginning of the lifecycle when a brand new product is being studied. Furthermore, social scientists use uniform distribution to represent a lack of knowledge. For example, in a simulation where distribution is not known, uniform random variates are often used. Uniform distribution is also used to describe the measurement error of some instruments or measuring systems. All of these factors (cf. [34]) explain the increasing interest in the choice of simple and computationally efficient tests for hypotheses about the uniform law of analyzed samples.

The aforesaid theoretical and practical importance of the statistic CRTE defined in (2) and the tests for uniformity provides a sufficient motivation to study and reveal some important properties of that statistic and use it for testing the uniformity.

The rest of the paper is organized as follows. In Section 2, we obtain some new findings of the CRTE. In Section 3, we propose the CRTE test statistic for uniformity and discuss some of its properties, including the property of stability. In Section 5, we propose the methods of finding the percentage points of CRTE. In addition, we estimate the percentage points of CRTE. In Section 6, we use a Monte Carlo simulation to carry out the power comparison of the uniformity of different tests against seven alternative distributions.

\section{Some Properties of CRTE}

In what follows, the symbols $\left(\frac{p}{n}\right),\left(\frac{d}{n}\right)$, and ( $\left.\frac{\text { a.s. }}{n}\right)$ stand for convergence in probability, convergence in distribution and almost surely, as $n \rightarrow \infty$. In this section, we derive some properties of the measure $C T_{\theta}(X)$, which is defined in (2).

Theorem 1. Let $X=\left(X_{1}, X_{2}, \ldots, X_{N}\right)$ be a random vector in $\mathbb{R}^{N}$. Furthermore, for all $1 \leq i \leq N$ and some $q>N$, let $X_{i} \in L^{q}$, i.e., $E\left[\left|X_{i}\right|^{q]}\right]<\infty$. Then, $C_{\theta}(X)<\infty, \theta>1$.

Proof. For all $0 \leq x \leq 1$ and $1 \neq \theta>0$, we can easily check that the function $g(x ; \theta):=$ $\frac{1}{\theta-1}\left(x-x^{\theta}\right)$ attains its maximum value $\theta^{\frac{\theta}{1-\theta}}$ at $x_{0}=\theta^{-\frac{1}{\theta-1}}$. Moreover,

$$
0 \leq g(x ; \theta) \leq \theta^{\frac{\theta}{1-\theta}} \leq 1,0 \leq x \leq 1,1 \neq \theta>0 .
$$

On the other hand, for each $0<p<1$, we are now going to prove the inequality

$$
g(x ; \theta)=\frac{1}{\theta-1}\left(x-x^{\theta}\right) \leq \theta^{\frac{\theta}{1-\theta}} \frac{x^{p}}{1-p^{\prime}}, 0 \leq x \leq 1, \theta>1 .
$$


Clearly, in view of the relation (3), the inequality (4) holds if $\mathcal{L}(x ; p):=\frac{x^{p}}{1-p} \geq 1$. Since, for $\mathcal{L}(x ; p)<1$, we obtain $x<(1-p)^{\frac{1}{p}}$, we consider the ratio $\frac{g(x ; \theta) \theta^{\frac{\theta}{\theta-1}}}{\mathcal{L}(x ; p)}$ in the interval $x<(1-p)^{\frac{1}{p}}$, where $\theta>1$. By using (3), we obtain

$$
\frac{g(x ; \theta) \theta^{\frac{\theta}{\theta-1}}}{\mathcal{L}(x ; p)} \leq \frac{1-p}{\theta-1}\left(x^{1-p}-x^{\theta-p}\right) \leq \frac{(1-p) x^{1-p}}{\theta-1} \leq \frac{1-p}{\theta-1}(1-p)^{\frac{1-p}{p}}=\frac{(1-p)^{\frac{1}{p}}}{\theta-1} .
$$

Thus, the inequality (4) holds if $\frac{(1-p)^{\frac{1}{p}}}{\theta-1} \leq 1$ or, equivalently, $\theta \geq 1+(1-p)^{\frac{1}{p}}$. On the other hand, since $p$ may be arbitrarily chosen in the interval $(0,1)$, we can choose it to be sufficiently close to 1 , in order that the inequality (4) be held for any $\theta>1$. This proves the inequality (4). It is worth mentioning that this inequality is not satisfied for $\theta<1$, in general, Figure 1 shows this fact.
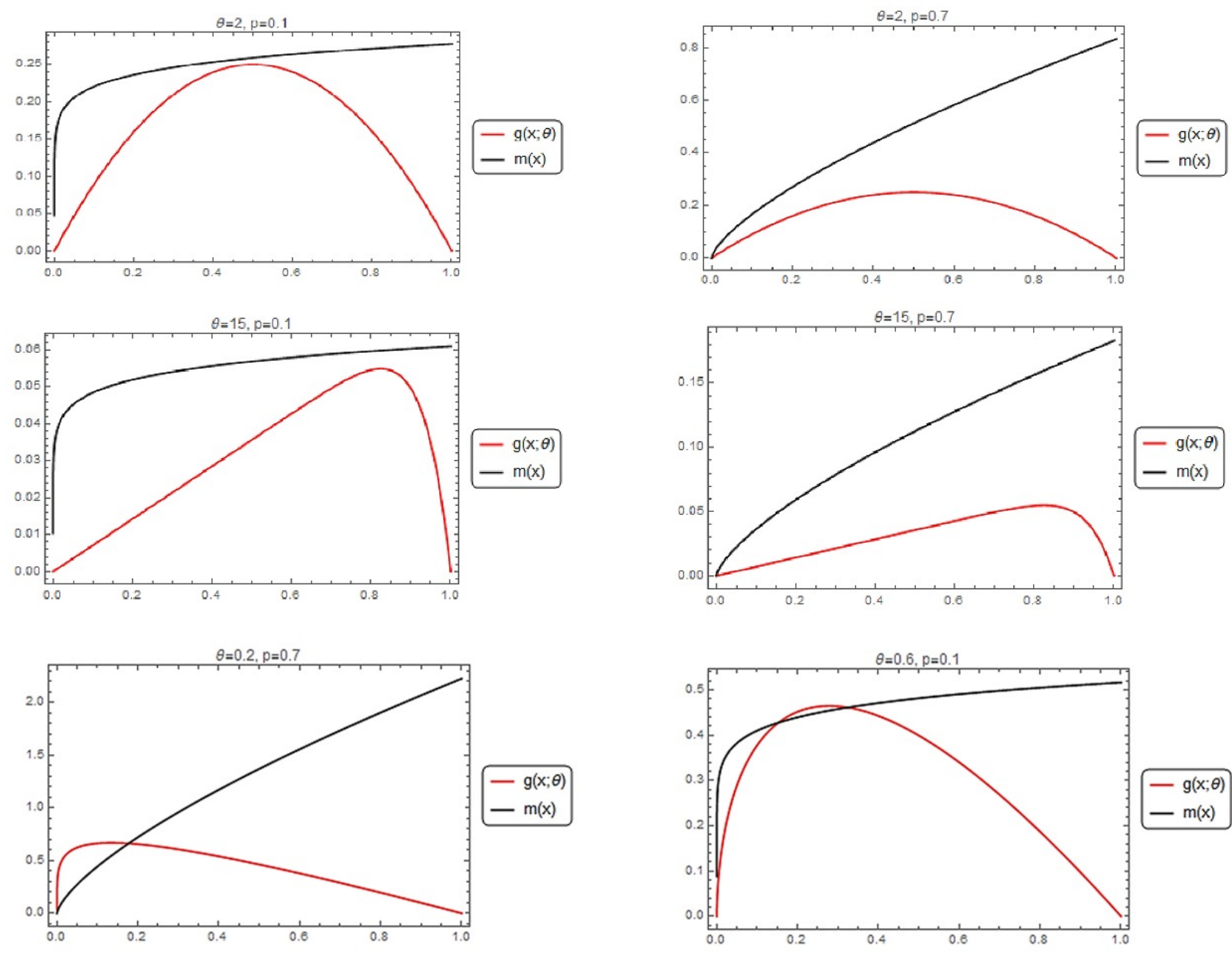

Figure 1. The relation between the functions $g(x ; \theta)$ and $m(x):=\theta^{\frac{\theta}{1-\theta}} \mathcal{L}(x ; p)$ with different values of $\theta$ and $0<p<1$. 
Now, for any $0<p<1$ and $1 \leq i \leq N$, we can use the inequality (4) to obtain

$$
\begin{aligned}
& \frac{1}{\theta-1}\left[P\left[\left|X_{i}\right|>x_{i}, 1 \leq i \leq N\right]-P^{\theta}\left[\left|X_{i}\right|>x_{i}, 1 \leq i \leq N\right]\right] \\
\leq & \frac{\theta^{\frac{\theta}{1-\theta}}}{1-p} P^{p}\left[\left|X_{i}\right|>x_{i}, 1 \leq i \leq N\right] \leq \frac{\theta^{\frac{\theta}{1-\theta}}}{1-p} \prod_{i=1}^{N} \bar{F}_{\left|X_{i}\right|}^{\frac{p}{N}}\left(x_{i}\right) .
\end{aligned}
$$

Integrating both sides of (5) over $\mathbb{R}_{+}^{N}=\left\{x_{i} \in \mathbb{R}^{N} ; x_{i} \geq 0\right\}$ and utilizing the Markov inequality, we obtain

$$
\begin{aligned}
C T_{\theta}(X) & \leq \frac{\theta^{\frac{\theta}{1-\theta}}}{1-p} \int_{\mathbb{R}_{+}^{N}} \prod_{i=1}^{N} \bar{F}_{\left|X_{i}\right|}^{\frac{p}{N}}\left(x_{i}\right) d x_{i}=\frac{\theta^{\frac{\theta}{1-\theta}}}{1-p} \prod_{i=1}^{N}\left\{\int_{0}^{\infty} \bar{F}_{\left|X_{i}\right|}^{\frac{p}{N}}\left(x_{i}\right) d x_{i}\right\} \\
& =\frac{\theta^{\frac{\theta}{1-\theta}}}{1-p} \prod_{i=1}^{N}\left\{\int_{0}^{1} \bar{F}_{\left|X_{i}\right|}^{\frac{p}{N}}\left(x_{i}\right) d x_{i}+\int_{1}^{\infty} \bar{F}_{\left|X_{i}\right|}^{\frac{p}{N}}\left(x_{i}\right) d x_{i}\right\} \\
& \leq \frac{\theta^{\frac{\theta}{1-\theta}}}{1-p} \prod_{i=1}^{N}\left\{1+\int_{1}^{\infty}\left[\frac{1}{x_{i}^{q}} E\left[\left|X_{i}\right|^{q}\right]\right]^{\frac{p}{N}} d x_{i}\right\},
\end{aligned}
$$

which is finite if $\frac{p q}{N}>1$. Thus, for any $q>N$, we can choose $p<1$ (sufficiently close to one) to satisfy $\frac{p q}{N}>1$ and the result follows.

Remark 1. For any $R V X$, it is well-known that the existence of $\operatorname{Var}(X)$ implies $X \in L^{2}$. Thus, the existence of $\operatorname{Var}(X)$ is a sufficient condition for $\mathrm{CT}_{\theta}(X)<\infty, \theta>1$.

Theorem 2 (Weak convergence). Let the sequence $X_{n}$ of $N$-dimensional random vectors converge in distribution to a random vector $X$. Furthermore, for all $n$, let $X_{n} \in L^{q}, q>N$. Then,

$$
\lim _{n \rightarrow+\infty} C T_{\theta}\left(X_{n}\right)=C T_{\theta}(X) .
$$

Proof. Since $X_{n} \underset{n}{\stackrel{d}{h}} X$, we have

$$
\lim _{n \rightarrow+\infty}\left(\bar{F}_{\left|X_{n}\right|}(x)-\bar{F}_{\left|X_{n}\right|}^{\theta}(x)\right)=\bar{F}_{|X|}(x)-\bar{F}_{|X|}^{\theta}(x), x \in \mathbb{R}_{+}^{N} .
$$

Meanwhile, from (4), we obtain

$$
\begin{aligned}
\frac{1}{\theta-1}\left(\bar{F}_{\left|X_{n}\right|}(x)-\bar{F}_{\left|X_{n}\right|}^{\theta}(x)\right) & \leq \frac{\theta^{\frac{\theta}{1-\theta}}}{1-p} \prod_{i=1}^{N} \bar{F}_{\left|X_{n_{i}}\right|}^{\frac{p}{N}}\left(x_{i}\right) \\
& \leq \frac{\theta^{\frac{\theta}{1-\theta}}}{1-p} \prod_{i=1}^{N}\left[I_{[0,1]}\left(x_{i}\right)+x_{i}^{-q} I_{[1, \infty)}\left(x_{i}\right) \mathrm{E}\left(\left|X_{n_{i}}\right|^{q}\right)\right]^{\frac{p}{N}},
\end{aligned}
$$

where $X_{n_{i}}$ is the $i$ th component of the random vector $X_{n}$ and $I_{A}(x)$ is the indicator function, i.e., $I_{A}(x)=1, x \in A, I_{A}(x)=0, x \notin A$. Therefore, if $\frac{q p}{N}>1,\left[\frac{1}{\theta-1}\left(\bar{F}_{\left|X_{n}\right|}(x)-\bar{F}_{\left|X_{n}\right|}^{\theta}(x)\right)\right]$ is bounded by an integrable function. Meanwhile, for any $q>N$, we can choose $p<1$ sufficiently close to one to satisfy $\frac{p q}{N}>1$. The use of the dominated convergence theorem completes the proof.

We show below that the measure $C T_{\theta}(X)$ dominates the differential entropy (1), which may exist when $X$ has density.

Theorem 3. Suppose that $X$ is a non-negative $R V$ with $C D F F_{X}(x)$; then,

$$
C T_{\theta}(X) \geq C(\theta) e^{S(X)}, 1 \neq \theta>0,
$$


where $C(\theta)=e^{\int_{0}^{1} \log \left(\frac{1}{\theta-1}\left(x-x^{\theta}\right)\right) d x}<\infty$, and $S(X)$ is the differential entropy defined in (1).

Proof. Utilizing the log-sum inequality, we obtain

$$
\int_{0}^{\infty} f_{X}(x) \log \left(\frac{f_{X}(x)}{\frac{1}{\theta-1}\left(\bar{F}_{X}(x)-\bar{F}_{X}^{\theta}(x)\right)}\right) d x \geq \log \frac{1}{\int_{0}^{\infty} \frac{1}{\theta-1}\left(\bar{F}_{X}(x)-\bar{F}_{X}^{\theta}(x)\right) d x}=-\log C T_{\theta}(X) .
$$

Furthermore, the left-hand side in (6) is derived as

$$
\int_{0}^{\infty} f_{X}(x) \log \left(\frac{f_{X}(x)}{\frac{1}{\theta-1}\left(\bar{F}_{X}(x)-\bar{F}_{X}^{\theta}(x)\right)}\right) d x=-S(X)-\int_{0}^{1} \log \left(\frac{1}{\theta-1}\left(x-x^{\theta}\right)\right) d x .
$$

Thus,

$$
\log C T_{\theta}(X) \geq S(X)+\int_{0}^{1} \log \left(\frac{1}{\theta-1}\left(x-x^{\theta}\right)\right) d x .
$$

The result follows after exponentiating both sides of (7) and using (3), where $C(\theta)=$ $e^{\int_{0}^{1} \ln \left(\frac{1}{\theta-1}\left(x-x^{\theta}\right)\right) d x} \leq \theta^{\frac{\theta}{1-\theta}}<1$ is finite. This completes the proof.

\section{Further Theoretical Aspects and Test Statistic}

To establish the test with a null hypothesis $H_{0}$ of uniformity, we need the following theorem.

Theorem 4. Let $X$ be a non-negative $R V$ with a continuous $C D F F$ with a support $[0,1]$. Then, $0 \leq C T_{\theta}(F) \leq \theta^{\frac{\theta}{1-\theta}}$. Moreover, the value $\frac{1}{2(1+\theta)}$ is uniquely attained by the uniform distribution $U(0,1)$ for all $1 \neq \theta>0$.

Proof. The proof of inequality $0 \leq C T_{\theta}(F) \leq \theta^{\frac{\theta}{1-\theta}}$ follows directly from (3). Meanwhile, using the strict concavity of $g(x ; \theta)=\frac{1}{\theta-1}\left(x-x^{\theta}\right)$, we obtain $C T_{\theta}($.$) as a concave function$ of distributions (with support $[0,1])$. Thus, $C T_{\theta}(F)=\frac{1}{2(1+\theta)}$ is uniquely acquired by the distribution $U(0,1)$. This completes the proof.

Let $X_{1}, X_{2}, \ldots, X_{n}$ be a random sample with a continuous CDF $F$ defined on $[0,1]$. Furthermore, let $X_{(1)} \leq X_{(2)} \leq \ldots \leq X_{(n)}$ be the corresponding order statistics. Clearly, we can suggest an estimator of $C T_{\theta}(F)$ by $C T_{\theta}\left(F_{n}\right)=\int_{0}^{\infty} g\left(\bar{F}_{n}(x) ; \theta\right) d x, 1 \neq \theta>0$, where $\bar{F}_{n}(x)=1-F_{n}(x)$ and $F_{n}(x)$ is the empirical $C D F$, which is given by

$$
F_{n}(x)=\sum_{i=1}^{n-1} \frac{i}{n} I_{\left[X_{(i)}, X_{(i+1)}\right)}(x)+I_{\left[X_{(n)}, \infty\right)}(x), x \in \mathbb{R} .
$$

Moreover, in order to get a consistent test of the hypothesis of uniformity, we propose the consistent statistic test

$$
\mathcal{R}_{n}(\theta)=\frac{1}{\theta-1} \sum_{i=1}^{n-1}\left(\left(1-\frac{i}{n}\right)-\left(1-\frac{i}{n}\right)^{\theta}\right)\left(X_{(i+1)}-X_{(i)}\right)=\sum_{i=1}^{n-1} A_{i} \Delta_{i}
$$

where $A_{i}=\frac{1}{\theta-1}\left(\left(1-\frac{i}{n}\right)-\left(1-\frac{i}{n}\right)^{\theta}\right), 1 \neq \theta>0$, and $\Delta_{i}=\left(X_{(i+1)}-X_{(i)}\right), i=1,2, \ldots$, $n-1$.

Theorem 5. The test based on the sample estimate $\mathcal{R}_{n}(\theta)$ is consistent.

Proof. From the Glivenko-Cantelli theorem, see Howard [35], we have $\sup _{t} \mid F_{n}(t)-$ $F(t) \mid \underset{n}{\stackrel{a . s .}{\rightarrow}}$ 0. Moreover, it is easily asserted that $C T_{\theta}\left(F_{n}\right) \underset{n}{\stackrel{a . s .}{h}} C T_{\theta}(F)$, which proves the theorem. 
Remark 2. Since $C T_{\theta}\left(F_{n}\right) \stackrel{p}{\rightarrow} C T_{\theta}(F)$, we obtain $\mathcal{R}_{n}(\theta) \stackrel{p}{n} \frac{1}{2(1+\theta)}$, under the null hypothesis $H_{0}$. On the other hand, under the alternative hypothesis (that $F$ is any continuous CDF defined on $[0,1]$, which is not the uniform) we have $\mathcal{R}_{n}(\theta) \stackrel{p}{\rightarrow} q$, where $q$ is a smaller or larger number than $\frac{1}{2(1+\theta)}$.

Theorem 6. Let the random sample $X_{1}, X_{2}, \ldots, X_{n}$ be drawn from an unknown continuous CDF $F$ defined on $[0,1]$. Then, $0 \leq \mathcal{R}_{n}(\theta) \leq \theta^{\frac{\theta}{1-\theta}}, 1 \neq \theta>0$.

Proof. In view of (3), we obtain

$$
0 \leq \mathcal{R}_{n}(\theta) \leq \sum_{i=1}^{n-1} \theta^{\frac{\theta}{1-\theta}} \Delta_{i}=\theta^{\frac{\theta}{1-\theta}}\left(X_{(n)}-X_{(1)}\right) \leq \theta^{\frac{\theta}{1-\theta}}
$$

This completes the proof.

Theorem 7. Under $H_{0}$, the mean and variance of $\mathcal{R}_{n}(\theta)$ are given, respectively, by

$$
E\left(\mathcal{R}_{n}(\theta)\right)=\frac{1}{n+1} \sum_{i=1}^{n-1} A_{i} \text {, and } \operatorname{Var}\left(\mathcal{R}_{n}(\theta)\right)=\frac{n}{(n+1)^{2}(n+2)} \sum_{i=1}^{n-1} A_{i}^{2} \text {. }
$$

Proof. Clearly, for any $i=1,2, \ldots, n-1$, the RV $\Delta_{i}$, based on the uniform distribution $U(0,1)$ has a beta distribution with parameter-vector $(1, n)$, written $\Delta_{i} \sim \operatorname{Beta}(. ; 1, n)$ (cf. [36]). This completes the proof.

Remark 3. Under $H_{0}$, we have $\lim _{n \rightarrow \infty} E\left(\mathcal{R}_{n}(\theta)\right)=\frac{1}{2(1+\theta)}$, and $\lim _{n \rightarrow \infty} \operatorname{Var}\left(\mathcal{R}_{n}(\theta)\right)=0$.

The critical region, which specifies the uniformity test, is defined by

$$
C T_{\theta}\left(F_{n}\right) \leq C T_{\theta, \frac{\alpha}{2}}^{\star}:=\text { lower, } \quad \text { or } \quad C T_{\theta}\left(F_{n}\right) \geq C T_{\theta, 1-\frac{\alpha}{2}}^{\star}:=\text { upper, }
$$

where $\alpha$ is the desired level of significance, and $C T_{\theta, \alpha}^{\star}$ is the $\alpha$-quantile of the asymptotic, or approximated, $\mathrm{CDF}$ of the test statistic $C T_{\theta}\left(F_{n}\right)$, under $H_{0}$.

\section{The Stability of CRTE}

The stability of measures of information has been studied by several works of literature, see [19,37-40]. Analogously, we define the stability of the CRTE as the following.

Definition 1. Let $X_{1}, X_{2}, \ldots, X_{n}$ be a random sample with a continuous CDF $F$ and $X_{1}^{\prime}, X_{2}^{\prime}, \ldots, X_{n}^{\prime}$ be any small deformation of $X_{1}, X_{2}, \ldots, X_{n}$. Then, the empirical CRTE is stable if $\forall \epsilon>0, \exists \delta>0$, and $\forall n \in \mathbb{Z}^{+}$, we have $\sum_{i=1}^{n}\left|X_{i}-X_{i}^{\prime}\right|<\delta \Rightarrow\left|C T_{\theta}\left(\bar{F}_{n}(X)\right)-C T_{\theta}\left(\bar{F}_{n}\left(X^{\prime}\right)\right)\right|<\epsilon$.

The next theorem gives a sufficient condition of the stability of the empirical CRTE.

Theorem 8. For any continuous $R V X$, the empirical CRTE is stable if $X$ is distributed on a finite interval.

Proof. Suppose that the RV $X$ is supported in the finite interval $[a, b]$ such that $a \geq 0$ and $b<\infty$. In view of (8), the empirical CRTE can be derived as

$$
C T_{\theta}\left(\bar{F}_{n}(X)\right)= \begin{cases}1-\frac{1}{n} \sum_{j=1}^{n} X_{j} & \theta=0, \\ \frac{1}{\theta-1} \sum_{j=1}^{n-1}\left(\bar{F}_{n}\left(X_{(j)}\right)-\bar{F}_{n}^{\theta}\left(X_{(j)}\right)\right) \Delta_{j}, & 1 \neq \theta>0 .\end{cases}
$$


For $\theta=0$, the stability of empirical CRTE is obvious. In brief, denote $g_{j}=g\left(\bar{F}_{n}\left(X_{(j)}\right) ; \theta\right)$, $g_{j}^{\prime}=g\left(\bar{F}_{n}\left(X_{(j)}^{\prime}\right) ; \theta\right)$, and $\Delta_{j}^{\prime}=X_{(j+1)}^{\prime}-X_{(j)}^{\prime}$. Thus, when $\sum_{j=1}^{n}\left|X_{j}-X_{j}^{\prime}\right|<\delta$, we obtain

$$
\begin{aligned}
& \left|C T_{\theta}\left(\bar{F}_{n}(X)\right)-C T_{\theta}\left(\bar{F}_{n}\left(X^{\prime}\right)\right)\right|=\left|\sum_{j=1}^{n-1} g_{j} \Delta_{j}-\sum_{j=1}^{n-1} g_{j}^{\prime} \Delta_{j}^{\prime}\right| \\
= & \left|\sum_{j=1}^{n-1}\left(g_{j}-g_{j}^{\prime}\right) \Delta_{j}+\sum_{j=1}^{n-1} g_{j}^{\prime}\left[\Delta_{j}-\Delta_{j}^{\prime}\right]\right| \leq \sum_{j=1}^{n-1}\left|g_{j}-g_{j}^{\prime}\right| \Delta_{j} \\
+ & \sum_{j=1}^{n-1} g_{j}^{\prime}\left[\left|\left(X_{(j+1)}-X_{(j+1)}^{\prime}\right)\right|+\left|\left(X_{(j)}-X_{(j)}^{\prime}\right)\right|\right] \leq \frac{\epsilon}{2(b-a)}\left(X_{(n)}-X_{(1)}\right)+2 \delta,
\end{aligned}
$$

where the second term in the second inequality in (10) is legitimated in view of (3). On the other hand, the first term in that inequality is legitimated from the fact that for any $x^{\prime}, x^{\prime \prime}$, and arbitrary small $\epsilon^{\star}>0, \exists \delta^{\star}>0$, such that $\left.\mid \bar{F}_{n}\left(x^{\prime}\right)-\bar{F}_{n}\left(x^{\prime \prime}\right)\right) \mid<\epsilon^{\star}$, whenever $\left|x^{\prime}-x^{\prime \prime}\right|<\delta^{\star}$ (cf. [19]), which implies $\left|g_{j}-g_{j}^{\prime}\right| \leq \frac{\epsilon}{2(b-a)}$, whenever, $\sum_{j=1}^{n}\left|X_{j}-X_{j}^{\prime}\right|<\delta$. Now, choose $\delta=\frac{\epsilon}{4}$, we obtain $\frac{\epsilon}{2(b-a)}\left(X_{(n)}-X_{(1)}\right)+2 \delta \leq \epsilon$. This completes the proof.

\section{Percentage Points of the Test Statistic}

In this section, we obtain the asymptotic distribution of $\mathcal{R}_{n}(\theta)$ under $H_{0}$. From (8), we can write $\mathcal{R}_{n}(\theta)=\sum_{i=1}^{n-1} T_{i}$, where $T_{i}=A_{i} \Delta_{i}, i=1,2, \ldots, n-1$, and $\Delta_{i} \sim \operatorname{Beta}(. ; 1, n)$. Thus, the RV $T_{i}$ has the PDF

$$
f_{T_{i}}(t)=\frac{n}{A_{i}}\left(1-\frac{t}{A_{i}}\right)^{n-1}, i=1,2, \ldots, n-1 .
$$

The mean and variance of $T_{i}$ are $\mu_{i}=\mathrm{E}\left(T_{i}\right)=A_{i} \mathrm{E}\left(\Delta_{i}\right)=\frac{A_{i}}{n+1}$ and $\sigma_{i}^{2}=\operatorname{Var}\left(T_{i}\right)=$ $A_{i}^{2} \operatorname{Var}\left(\Delta_{i}\right)=\frac{n A_{i}^{2}}{(n+1)^{2}(n+2)}$, respectively. By using the Lyapunov central limit theorem (cf. Billingsley [41]), we obtain $\frac{\sum_{i=1}^{n-1}\left(T_{i}-\mu_{i}\right)}{\sqrt{\sum_{i=1}^{n-1} \sigma_{i}^{2}}}=\frac{\mathcal{R}_{n}(\theta)-\mathrm{E}\left(\mathcal{R}_{n}(\theta)\right)}{\sqrt{\operatorname{Var}\left(\mathcal{R}_{n}(\theta)\right)}} \stackrel{d}{n} Z$, where $Z$ is the standard normal RV. Therefore, under $H_{0}$, the percentage point ( $\alpha$-quantile) $C T_{\theta, \alpha}^{\star}$ is estimated for large $n$ by using the asymptotic normality of $\mathcal{R}_{n}(\theta)$ as follows

$$
\widehat{C T}_{\theta, \alpha}^{\star}=\mathrm{E}\left(\mathcal{R}_{n}(\theta)\right)+\sqrt{\operatorname{Var}\left(\mathcal{R}_{n}(\theta)\right)} Z_{\alpha},
$$

where $Z_{\alpha}$ is the quantile $(\alpha \times 100)$ of the standard normal distribution $\Phi_{Z}($.$) .$

Johannesson and Giri [42] suggested an approximation of the CDF of the linear combination of the finite number of beta RVs. Noughabi [29] utilized this result to approximate the percentage points of the CRE for finite $n$. By following a similar method, an approximation of $\mathcal{R}_{n}(\theta)$ for finite $n$ can be obtained as follows:

$$
\mathcal{R}_{n}(\theta) \approx\left(\sum_{i=1}^{n-1} A_{i}\right) \eta
$$

where the $\mathrm{RV} \eta$ has the beta distribution $\operatorname{Bet} a(. ; a, b)$ with

$$
a=\frac{(n+2)\left(\sum_{i=1}^{n-1} A_{i}\right)^{2}}{(n+1)\left(\sum_{i=1}^{n-1} A_{i}^{2}\right)}-\frac{1}{n+1}, \text { and } b=\frac{n}{n+1}\left(\frac{(n+2)\left(\sum_{i=1}^{n-1} A_{i}\right)^{2}}{\sum_{i=1}^{n-1} A_{i}^{2}}-1\right) .
$$


According to (12), the mean and variance of $\mathcal{R}_{n}(\theta)$ are given, respectively, by

$$
\mathrm{E}\left(\mathcal{R}_{n}(\theta)\right)=\left(\sum_{i=1}^{n-1} A_{i}\right) \frac{a}{a+b}, \text { and } \operatorname{Var}\left(\mathcal{R}_{n}(\theta)\right)=\left(\sum_{i=1}^{n-1} A_{i}\right)^{2} \frac{a b}{(a+b)^{2}(a+b+1)} .
$$

Using this approximation of $\mathcal{R}_{n}(\theta)$, the quantiles of order $\frac{\alpha}{2}$ and $1-\frac{\alpha}{2}$ of the approximated $\mathrm{CDF}$ of the test statistic $C T_{\theta}\left(F_{n}\right)$ under $H_{0}$ are given, respectively, by

$$
\text { lower }:=\left(\sum_{i=1}^{n-1} A_{i}\right) \text { Beta }^{-1}\left(\frac{\alpha}{2} ; a, b\right), \text { and upper }:=\left(\sum_{i=1}^{n-1} A_{i}\right) \text { Beta }^{-1}\left(1-\frac{\alpha}{2} ; a, b\right),
$$

where $\operatorname{Bet}^{-1}(. ; a, b)$ is the quantile function of the beta distribution, Beta $(. ; a, b)$, and the parameter-vector $(a, b)$ is defined in (13).

\section{Percentage Points}

We generated 50,000 samples of sizes $n=10,20,30,40,50,70$, and 100 from $U(0,1)$. Utilizing (8), the test statistic $\mathcal{R}_{n}(\theta)$ was estimated by the empirical CRTE for each sample. Moreover, we can see that $C T_{0.1}(U)=0.4545, C T_{0.5}(U)=0.3333, C T_{0.9}(U)=0.2631$, $C T_{2}(U)=\frac{1}{6}, C T_{5}(U)=\frac{1}{12}$, and $C T_{10}(U)=\frac{1}{22}$, where $C T_{\theta}(U)$ is the CRTE of the CDF $U(0,1)$. Consequently, for $\mathcal{R}_{n}(\theta)$, we present the percentage points of the Monte Carlo method, asymptotic normality, and beta approximation by using (9), (11), and (14), respectively. Table 1 shows that as $n$ increased, the difference between percentage points (upper-lower) decreased. Furthermore, the Monte Carlo approach was more accurate than the other two methods for $\mathcal{R}_{n}(\theta)$, because it had almost minimum differences between percentage points.

Figures 2 and 3 depict the empirical PDFs of the test statistics via Monte Carlo samples for $n=10,20,30,50$, and 100, $\theta=0.1,0.5$, and 0.9 , and $n=10,20,30,50$, and 100, $\theta=2,5$, and 10, respectively. It is noted that the means of the empirical PDFs of $\mathcal{R}_{n}(\theta)$ became nearer to the exact values $\left(C T_{\theta}(U)\right)$ as $n$ increased, which indicates that the bias and variance decreased with an increase in $n$. Moreover, the six corresponding figures to $\theta=0.1,0.5,0.9,2,5$, and 10 in Figures 2 and 3 reveal that the improvement in the bias and variance does not depend almost on $\theta$. 

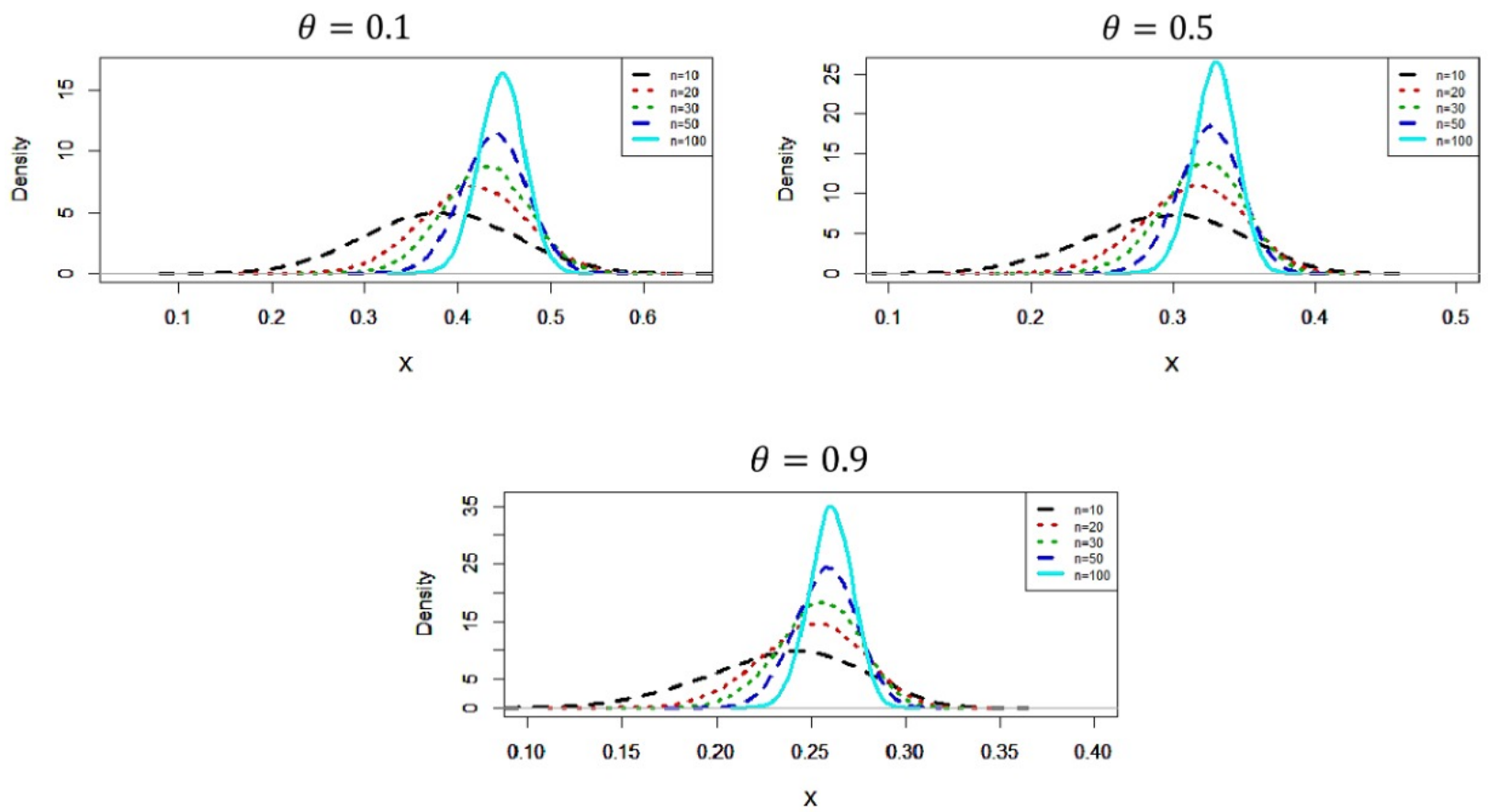

Figure 2. The estimated PDFs of $\mathcal{R}_{n}(\theta)$ under $U(0,1)$, for $0<\theta<1$.
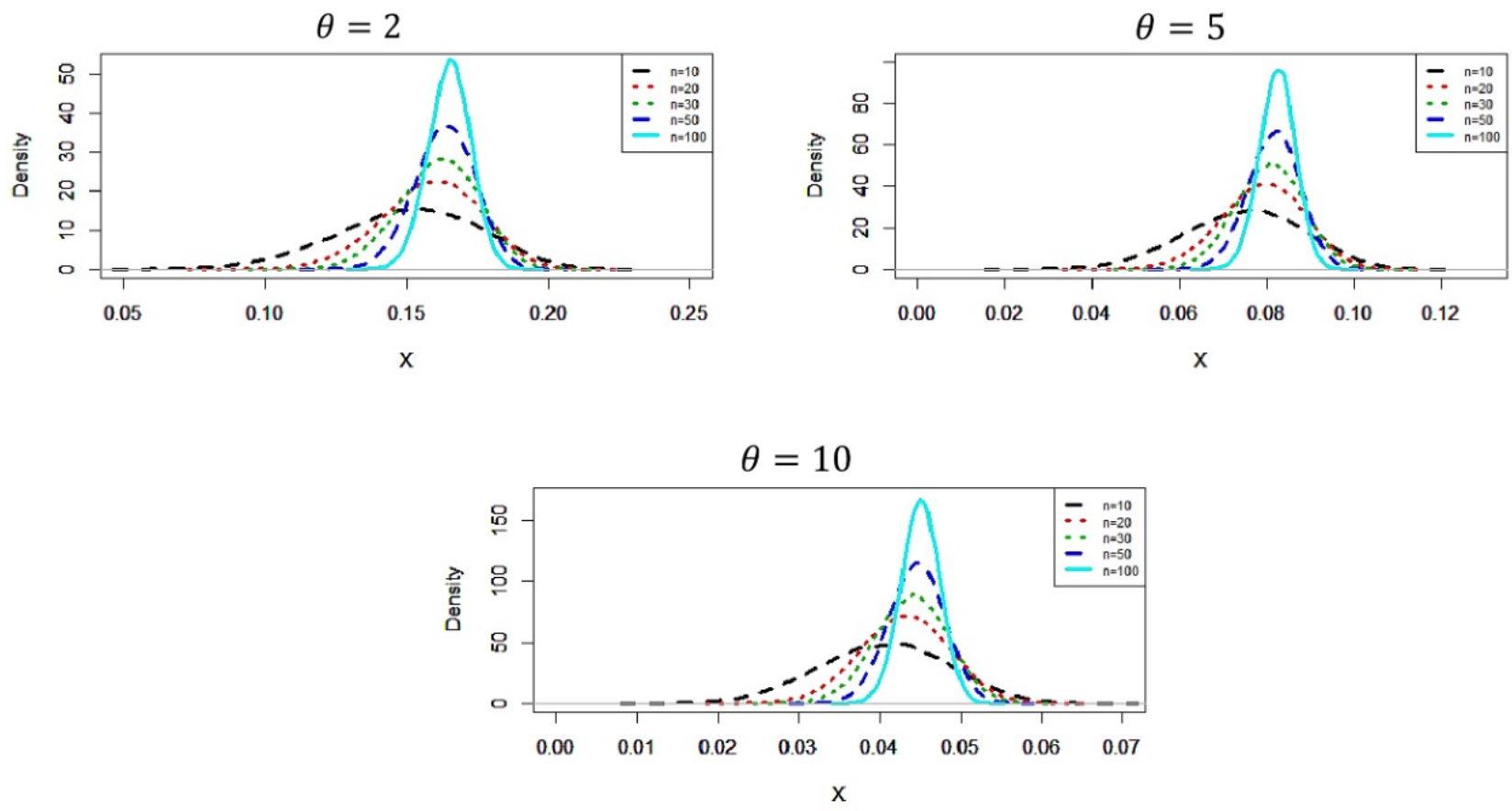

Figure 3. The estimated PDFs of $\mathcal{R}_{n}(\theta)$ under $U(0,1)$, for $\theta>1$. 
Table 1. Percentage points of the suggested test statistic $\mathcal{R}_{n}(\theta)$ at level $\alpha=0.05$.

\begin{tabular}{|c|c|c|c|c|c|c|c|}
\hline \multirow{3}{*}{$n$} & \multirow{3}{*}{$\theta$} & \multicolumn{6}{|c|}{$\mathcal{R}_{n}(\theta)$} \\
\hline & & \multicolumn{2}{|c|}{ Normal Approximation } & \multicolumn{2}{|c|}{ Beta Approximation } & \multicolumn{2}{|c|}{ Monte Carlo Method } \\
\hline & & Lower & Upper & Lower & Upper & Lower & Upper \\
\hline \multirow[t]{6}{*}{10} & 0.1 & 0.1285 & 0.6311 & 0.1682 & 0.6655 & 0.2261 & 0.5302 \\
\hline & 0.5 & 0.1059 & 0.4777 & 0.1341 & 0.5023 & 0.1809 & 0.3872 \\
\hline & 0.9 & 0.0873 & 0.3815 & 0.1093 & 0.4007 & 0.1486 & 0.3056 \\
\hline & 2 & 0.05609 & 0.2439 & 0.07006 & 0.2561 & 0.0966 & 0.1956 \\
\hline & 5 & 0.0269 & 0.1226 & 0.0342 & 0.12902 & 0.0468 & 0.1002 \\
\hline & 10 & 0.01407 & 0.066908 & 0.01818 & 0.0704 & 0.0247 & 0.0554 \\
\hline \multirow[t]{6}{*}{20} & 0.1 & 0.2163 & 0.6167 & 0.2399 & 0.6381 & 0.3059 & 0.5228 \\
\hline & 0.5 & 0.1683 & 0.4581 & 0.1847 & 0.4731 & 0.2381 & 0.3781 \\
\hline & 0.9 & 0.1351 & 0.3631 & 0.1479 & 0.3748 & 0.1919 & 0.2976 \\
\hline & 2 & 0.0856 & 0.2309 & 0.0938 & 0.2384 & 0.12201 & 0.19017 \\
\hline & 5 & 0.04208 & 0.11614 & 0.04633 & 0.12001 & 0.0599 & 0.0967 \\
\hline & 10 & 0.022602 & 0.0635 & 0.0249 & 0.0657 & 0.0321 & 0.0534 \\
\hline \multirow[t]{6}{*}{30} & 0.1 & 0.2591 & 0.5993 & 0.2757 & 0.6147 & 0.3391 & 0.5145 \\
\hline & 0.5 & 0.1979 & 0.4425 & 0.2094 & 0.4532 & 0.2608 & 0.3723 \\
\hline & 0.9 & 0.1578 & 0.35001 & 0.1668 & 0.3583 & 0.2088 & 0.2933 \\
\hline & 2 & 0.0998 & 0.2223 & 0.1056 & 0.2276 & 0.1321 & 0.1868 \\
\hline & 5 & 0.0493 & 0.1117 & 0.0523 & 0.1145 & 0.06506 & 0.09504 \\
\hline & 10 & 0.02664 & 0.06113 & 0.02831 & 0.0626 & 0.03505 & 0.0524 \\
\hline \multirow[t]{6}{*}{40} & 0.1 & 0.2853 & 0.5859 & 0.2982 & 0.5979 & 0.3581 & 0.5098 \\
\hline & 0.5 & 0.2159 & 0.4313 & 0.2248 & 0.4396 & 0.2733 & 0.3688 \\
\hline & 0.9 & 0.1717 & 0.3408 & 0.1786 & 0.3473 & 0.2178 & 0.2904 \\
\hline & 2 & 0.10863 & 0.2163 & 0.11305 & 0.2205 & 0.1378 & 0.1847 \\
\hline & 5 & 0.0537 & 0.10869 & 0.05608 & 0.1108 & 0.0679 & 0.0938 \\
\hline & 10 & 0.0291 & 0.0594 & 0.0304 & 0.0606 & 0.0367 & 0.0516 \\
\hline \multirow[t]{6}{*}{50} & 0.1 & 0.3034 & 0.5755 & 0.3139 & 0.5853 & 0.3704 & 0.5052 \\
\hline & 0.5 & 0.2284 & 0.4229 & 0.2356 & 0.4297 & 0.2811 & 0.3661 \\
\hline & 0.9 & 0.1813 & 0.33405 & 0.1869 & 0.3393 & 0.2239 & 0.2883 \\
\hline & 2 & 0.1146 & 0.2119 & 0.1182 & 0.2153 & 0.1411 & 0.1834 \\
\hline & 5 & 0.0568 & 0.1064 & 0.0587 & 0.1082 & 0.0697 & 0.0929 \\
\hline & 10 & 0.03082 & 0.0582 & 0.0318 & 0.0592 & 0.0377 & 0.05111 \\
\hline \multirow[t]{6}{*}{70} & 0.1 & 0.3273 & 0.5604 & 0.3349 & 0.5676 & 0.3853 & 0.4993 \\
\hline & 0.5 & 0.2448 & 0.41109 & 0.25005 & 0.41608 & 0.2907 & 0.3617 \\
\hline & 0.9 & 0.19403 & 0.3245 & 0.1981 & 0.3284 & 0.2309 & 0.28504 \\
\hline & 2 & 0.1227 & 0.2058 & 0.1253 & 0.2083 & 0.1459 & 0.1811 \\
\hline & 5 & 0.06094 & 0.1033 & 0.0623 & 0.1046 & 0.0722 & 0.0916 \\
\hline & 10 & 0.03308 & 0.056509 & 0.0338 & 0.0572 & 0.03908 & 0.0503 \\
\hline \multirow[t]{6}{*}{100} & 0.1 & 0.3486 & 0.5456 & 0.35404 & 0.5508 & 0.3982 & 0.4943 \\
\hline & 0.5 & 0.2594 & 0.3997 & 0.2631 & 0.4033 & 0.29883 & 0.3581 \\
\hline & 0.9 & 0.2054 & 0.3155 & 0.2083 & 0.3183 & 0.2373 & 0.2822 \\
\hline & 2 & 0.1299 & 0.20008 & 0.1317 & 0.2018 & 0.1497 & 0.1792 \\
\hline & 5 & 0.0646 & 0.1003 & 0.0655 & 0.1013 & 0.0741 & 0.0904 \\
\hline & 10 & 0.0351 & 0.0548 & 0.0356 & 0.0553 & 0.04018 & 0.0496 \\
\hline
\end{tabular}

\section{Power Analysis}

In this section, we examine the power test of the Monte Carlo method under alternative distributions. The power of $\mathcal{R}_{n}(\theta)$ is estimated by the proportion of the generated samples that are in the critical region. Under seven alternative distributions, the power of $\mathcal{R}_{n}(\theta)$ is calculated by the Monte Carlo method for the generated 50,000 samples each of size $n=10,20$, and 40 . The alternative CDFs introduced by Stephens [27] in the power study of uniformity tests are 


$$
\begin{aligned}
& A_{l}: F(y)=1-(1-y)^{l}, \quad 0 \leq y \leq 1, l=1.5,2, \\
& B_{l}: F(y)= \begin{cases}2^{l-1} y^{l}, \quad 0 \leq y \leq 0.5, \\
1-2^{l-1}(1-y)^{l}, \quad 0.5 \leq y \leq 1, l=1.5,2,3,\end{cases} \\
& C_{l}: F(y)= \begin{cases}0.5-2^{l-1}(0.5-y)^{l}, & 0 \leq y \leq 0.5, \\
0.5+2^{l-1}(y-0.5)^{l}, & 0.5 \leq y \leq 1, l=1.5,2 .\end{cases}
\end{aligned}
$$

On the report of Stephens [27], the family $A_{l}$ gives points nearer to zero than predictable under the hypothesis of uniformity and is interpreted as a change in the mean, the family $B_{l}$ gives points near 0.5 and is interpreted as a change toward a smaller variance, and the family $C_{l}$ shows two clusters close to 0,1 and is interpreted as a change toward a larger variance.

In Table 2, we recorded the power values of the proposed test statistics $\mathcal{R}_{n}(\theta)$, Kolmogorov-Smirnov (K-S), Kuiper (V), Cramer-von Mises $\left(W^{2}\right)$, Watson $\left(U^{2}\right)$, and Anderson-Darling $\left(A^{2}\right)$, for $n=10,20$, and 40 , and $\alpha=0.05$. From Table 2, we can draw the following conclusions:

1. For a fixed $\theta$ and as $n$ increases, we see that the power of $\mathcal{R}_{n}(\theta)$ increases.

2. For the alternatives $B_{l}$ and $C_{l}$, the power of $\mathcal{R}_{n}(\theta)$ increases and gives better performance against the other tests when $\theta$ tends to $1(\theta \rightarrow 1)$.

3. For the alternative $A_{l}$, when $n$ and $\theta$ increases, the power of $\mathcal{R}_{n}(\theta)$ increases and gives

\begin{tabular}{|c|c|c|c|c|c|c|c|c|c|c|}
\hline \multirow{2}{*}{$n$} & \multirow{2}{*}{ Alternative } & \multicolumn{4}{|c|}{$\mathcal{R}_{n}(\theta)$} & \multirow{2}{*}{ K-S } & \multirow{2}{*}{$\mathbf{V}$} & \multirow{2}{*}{$W^{2}$} & \multirow{2}{*}{$U^{2}$} & \multirow{2}{*}{$A^{2}$} \\
\hline & & $\theta=0.1$ & $\theta=0.9$ & $\theta=2$ & $\theta=10$ & & & & & \\
\hline \multirow[t]{7}{*}{10} & $A_{1.5}$ & 0.079 & 0.06656 & 0.08072 & 0.10558 & 0.12616 & 0.0756 & 0.1456 & 0.07776 & 0.1877 \\
\hline & $A_{2}$ & 0.11404 & 0.12564 & 0.18706 & 0.27216 & 0.30298 & 0.1631 & 0.3551 & 0.16308 & 0.4761 \\
\hline & $B_{1.5}$ & 0.095 & 0.11868 & 0.13762 & 0.10504 & 0.07352 & 0.0971 & 0.0741 & 0.1017 & 0.1349 \\
\hline & $B_{2}$ & 0.22202 & 0.30572 & 0.3577 & 0.25458 & 0.1184 & 0.2307 & 0.1104 & 0.2481 & 0.3269 \\
\hline & $B_{3}$ & 0.5349 & 0.72444 & 0.7997 & 0.61728 & 0.2424 & 0.5394 & 0.2154 & 0.5699 & 0.72308 \\
\hline & $C_{1.5}$ & 0.0804 & 0.11834 & 0.13002 & 0.1076 & 0.0342 & 0.0974 & 0.0239 & 0.1031 & 0.0222 \\
\hline & $C_{2}$ & 0.12666 & 0.24366 & 0.27896 & 0.18578 & 0.0402 & 0.2333 & 0.01114 & 0.2475 & 0.00924 \\
\hline \multirow[t]{7}{*}{20} & $A_{1.5}$ & 0.10758 & 0.07272 & 0.13104 & 0.24056 & 0.2179 & 0.1226 & 0.25208 & 0.1225 & 0.3235 \\
\hline & $A_{2}$ & 0.15218 & 0.1876 & 0.39546 & 0.63934 & 0.5616 & 0.3486 & 0.6241 & 0.3358 & 0.7538 \\
\hline & $B_{1.5}$ & 0.13084 & 0.25316 & 0.30462 & 0.18358 & 0.0869 & 0.1634 & 0.0781 & 0.1786 & 0.1774 \\
\hline & $B_{2}$ & 0.3462 & 0.66858 & 0.75146 & 0.50604 & 0.1849 & 0.4647 & 0.162 & 0.5067 & 0.52802 \\
\hline & $B_{3}$ & 0.76144 & 0.9852 & 0.99594 & 0.9319 & 0.4588 & 0.8711 & 0.4615 & 0.8978 & 0.93998 \\
\hline & $C_{1.5}$ & 0.0897 & 0.19596 & 0.2244 & 0.14142 & 0.0509 & 0.1621 & 0.02406 & 0.1791 & 0.0213 \\
\hline & $C_{2}$ & 0.1361 & 0.42338 & 0.53846 & 0.2519 & 0.1162 & 0.4633 & 0.0462 & 0.5048 & 0.0338 \\
\hline \multirow[t]{7}{*}{40} & $A_{1.5}$ & 0.20048 & 0.08398 & 0.24962 & 0.51094 & 0.3144 & 0.18002 & 0.366 & 0.1721 & 0.4498 \\
\hline & $A_{2}$ & 0.2663 & 0.29604 & 0.72538 & 0.94942 & 0.7522 & 0.5447 & 0.8105 & 0.5071 & 0.8973 \\
\hline & $B_{1.5}$ & 0.1668 & 0.5149 & 0.61588 & 0.34912 & 0.1021 & 0.2477 & 0.0873 & 0.2667 & 0.2281 \\
\hline & $B_{2}$ & 0.4824 & 0.95894 & 0.98322 & 0.8408 & 0.2706 & 0.6695 & 0.25108 & 0.7076 & 0.7002 \\
\hline & $B_{3}$ & 0.90634 & 1 & 1 & 0.99952 & 0.6701 & 0.97506 & 0.7237 & 0.9819 & 0.99104 \\
\hline & $C_{1.5}$ & 0.10134 & 0.32746 & 0.4469 & 0.18216 & 0.07 & 0.2492 & 0.0303 & 0.2678 & 0.0271 \\
\hline & $C_{2}$ & 0.1511 & 0.64696 & 0.87082 & 0.32854 & 0.2077 & 0.6711 & 0.1258 & 0.7111 & 0.1105 \\
\hline
\end{tabular}
a better performance than the other tests.

Table 2. Power estimates of the tests at the level $\alpha=0.05$.

\section{Conclusions}

Some novel properties of the CRTE quantity were presented such as sufficient conditions for the CRTE to be finite, the weak convergence of the CRTE, the connections between the CRTE, the CRE, and classic differential entropy, and the stability of the empirical CRTE. Furthermore, for the CDFs with support $[0,1]$, we exhibited that the value of $C T_{\theta}$ was within $\left[0, \theta^{\frac{\theta}{1-\theta}}\right]$. Moreover, the test of uniformity was proposed by calculating the percentage points and power analysis of $C T_{\theta}$. In addition, for $C T_{\theta}$, we obtained the percentage 
points by using the Monte Carlo method via simulation and normality asymptotic, as well as the beta approximation. A power comparison was performed between the CRTE and other tests, where, by changing the value of $\theta$, we indicated when the test had higher and lower power compared with the other tests.

When we talk about the prospects for future research, we consider here two problems. The first one is to extend the result of this work to a multivariate version of the entropy measures; see, for example, the Formulas (4), (5), and (6) of [43], as a starting point for that future work. The second future research goal is to apply the proposed test to a recent real-world dataset to help solve one of society's practical concerns.

Author Contributions: The authors contributed equally to the paper. All authors have read and agreed to the published version of the manuscript.

Funding: This research received no external funding.

Institutional Review Board Statement: Not applicable.

Informed Consent Statement: Not applicable.

Data Availability Statement: The simulated data used to support the findings of this study are included within the article.

Acknowledgments: The authors are grateful to Mata Wang and the four anonymous reviewers for their careful and diligent reading, which improved the readability and presentation substantially.

Conflicts of Interest: The authors declare no conflict of interest.

\section{References}

1. Shannon, C.E. A mathematical theory of communication. Bell. Syst. Tech. J. 1948, 27, 379-423. [CrossRef]

2. Li, Z.; Li, W.; Liu, R. Applications of entropy principles in power systems: A survey. In Proceedings of the 2005 IEEE/PES Transmission \& Distribution Conference \& Exposition: Asia and Pacific, Dalian, China, 15-18 August 2005; pp. 1-4.

3. Schmid, R.; Miah, A.M.; Sapunov, V.N. A new table of the thermodynamic quantities of ionic hydration: Values and some applications (enthalpy-entropy compensation and Born radii). Phys. Chem. Chem. Phys. 2000, 2, 97-102. [CrossRef]

4. Song, Y.; Fu, Q.; Wang, Y.F.; Wang, X. Divergence-based cross entropy and uncertainty measures of Atanassov's intuitionistic fuzzy sets with their application in decision making. Appl. Soft Comput. 2019, 84, 105703. [CrossRef]

5. Gu, R. Multiscale Shannon entropy and its application in the stock market. Physica A 2017, 484, 215-224. [CrossRef]

6. Zhou, R.; Cai, R.; Tong, G. Applications of entropy in finance: A review. Entropy 2013, 15, 4909-4931. [CrossRef]

7. Rao, M.; Chen, Y.; Vemuri, B.C.; Wang, F. Cumulative residual entropy: A new measure of information. IEEE Trans. Inf. Theory 2004, 50, 1220-1228. [CrossRef]

8. Wang, F.; Vemuri, B.C. Non-rigid multi-model image registration using cross-cumulative residual entropy. Int. J. Comp. Vision 2007, 74, 201-215. [CrossRef]

9. Havrda, J.; Charvat, F. Quantification method of classification process: Concept of structural $\alpha$-entropy. Kybernetika 1967, 3, 30-35.

10. Tsallis, C. Possible generalization of Boltzmann-Gibbs statistics. J. Stat. Phys. 1988, 52, 479-487. [CrossRef]

11. Sati, M.M.; Gupta, N. Some characterization results on dynamic cumulative residual Tsallis entropy. J. Probab. Stat. 2015, 2015, 694203. [CrossRef]

12. Rajesh, G.; Sunoj, S.M. Some properties of cumulative Tsallis entropy of order $\alpha$. Stat. Pap. 2019, 60, 933-943. [CrossRef]

13. Mohamed, M.S. On cumulative Tsallis entropy and its dynamic past version. Indian J. Pure Appl. Math. 2020, 51, 1903-1917. [CrossRef]

14. Abd Elgawad, M.A.; Alawady, M.A.; Barakat, H.M.; Xiong, S. Concomitants of generalized order statistics from Huang-Kotz Farlie-Gumbel-Morgenstern bivariate distribution: Some information measures. Bull. Malays. Math. Sci. Soc. 2020, 43, 2627-2645. [CrossRef]

15. Abd Elgawad, M.A.; Barakat, H.M.; Xiong, S.; Alyami, S.A. Information measures for generalized order statistics and their concomitants under general framework from Huang-Kotz FGM bivariate distribution. Entropy 2021, 23, 335. [CrossRef]

16. Alawady, M.A.; Barakat, H.M.; Abd Elgawad, M.A. Concomitants of generalized order statistics from bivariate Cambanis family of distributions under a general setting. Bull. Malays. Math. Sci. Soc. 2021, 44, 3129-3159. [CrossRef]

17. Barakat, H.M.; Husseiny, I.A. Some information measures in concomitants of generalized order statistics under iterated FarlieGumbel-Morgenstern bivariate type. Quaest. Math. 2021, 44, 581-598. [CrossRef]

18. Park, S. Information measure in terms of the hazard function and its estimate. Entropy 2021, 23, 298. [CrossRef]

19. Xiong, H.; Shang, P.; Zhang, Y. Fractional cumulative residual entropy. Comm. Nonlin. Sci. Num. Simul. 2019, 78, 104879. [CrossRef] 
20. Zhang, Y.; Shang, P.; He, J.; Xiong, H. Cumulative Tsallis entropy based on power spectrum of financial time series. Chaos 2019, 29, 103-118. [CrossRef]

21. Irshad, M.R.; Maya, R.; Buono, F.; Longobardi, M. Kernel estimation of cumulative residual Tsallis entropy and its dynamic version under $\rho$-mixing dependent data. Entropy 2022, 24, 9. [CrossRef]

22. Mohamed, M.S. On cumulative residual Tsallis entropy and its dynamic version of concomitants of generalized order statistics. Commun. Stat. Theory Methods 2020. [CrossRef]

23. Mohamed, M.S.; Abdulrahman, A.T.; Almaspoor, Z.; Yusuf, M. Ordered variables and their concomitants under extropy via COVID-19 data application. Complexity 2021, 2021, 114. [CrossRef]

24. Toomaj, A.; Atabay, H.A. Some new findings on the cumulative residual Tsallis entropy. J. Comput. Appl. Math. 2021, $400,113669$. [CrossRef]

25. Mohamed, M.S. A measure of inaccuracy in concomitants of ordered random variables under Farlie-Gumbel-Morgenstern family Filomat 2019, 33, 4931-4942. [CrossRef]

26. Mohamed, M.S. Some new findings on the survival Rényi entropy and application of COVID-19 data. Results Phys. 2021, 31, 104966. [CrossRef] [PubMed]

27. Stephens, M.A. EDF statistics for goodness of fit and some comparisons. J. Am. Stat. Assoc. 1974, 69, 730-737. [CrossRef]

28. Dudewicz, E.J.; Van der Meulen, E.C. Entropy-based tests of uniformity. J. Am. Stat. Assoc. 1981, 76, 967-974. [CrossRef]

29. Noughabi, H.A. Cumulative residual entropy applied to testing uniformity. Commun. Stat. Theory Methods 2020, 50, 1811339. [CrossRef]

30. Mohamed, M.S.; Barakat, H.M.; Alyami, S.A.; Abd Elgawad, M.A. Fractional entropy-based test of uniformity with power comparisons. J. Math. 2021, 2021, 5331260. [CrossRef]

31. Anastasiadis, A. Special Issue: Tsallis Entropy. Entropy 2012, 14, 174-176. [CrossRef]

32. Cartwright, J. Roll over, Boltzmann. Phys. World 2014, 27, 31-35. [CrossRef]

33. Wanke, P. The uniform distribution as a first practical approach to new product inventory management. Int. J. Prod. Econ. 2008, 114, 811-819. [CrossRef]

34. Blinov, P.Y.; Lemeshko, B.Y. A review of the properties of tests for uniformity. In Proceedings of the 201412 th International Conference on Actual Problems of Electronic Instrument Engineering, Novosibirsk, Russia, 2-4 October 2014. [CrossRef]

35. Howard, G.T. A generalization of the Glivenko-Cantelli theorem. Ann. Math. Stat. 1959, 30, 828-830. [CrossRef]

36. Arnold, B.C.; Balakrishnan, N.; Nagaraja, H.N. A First Course in Order Statistics; Wiley: New York, NY, USA, 1992.

37. Abe, S. Stability of Tsallis entropy and instabilities of Renyi and normalized Tsallis entropies: A basis for q-exponential distributions. Phys. Rev. E 2002, 66, 046134. [CrossRef] [PubMed]

38. Abe, S.; Kaniadakis, G.; Scarfone, A.M. Stabilities of generalized entropies. J. Phys. A Math. Gen. 2004, 37, 10513. [CrossRef]

39. Lesche, B. Instabilities of Renyi entropies. J. Stat. Phys. 1982, 27, 419-422. [CrossRef]

40. Ubriaco, M.R. Entropies based on fractional calculus. Phys. Lett. A 2009, 373, 2516-2519. [CrossRef]

41. Billingsley, P. Probability and Measure; John Wiley \& Sons: New York, NY, USA, 2008.

42. Johannesson, B.; Giri, N. On approximations involving the beta distribution. Commun. Stat. Simul. Comput. 1995, 24, 489-503. [CrossRef]

43. Mesiar, R.; Sheikhi, A. Nonlinear random forest classification, a copula-based approach. Appl. Sci. 2021, 11, 7140. [CrossRef] 